\title{
Sensor fusion for coastal waters surveillance
}

\author{
Bharat Doshi, Lotfi Benmohamed, Phil Chimento, I-Jeng Wang \\ Johns Hopkins University/Applied Physics Lab,11100 Johns Hopkins Road, Laurel, MD 20723;
}

\begin{abstract}
We describe a large sensor field whose mission is to protect coastal waters by detecting objects like submarines. The system is buoy-based and distributed over a littoral area. The opportunities for detection are short and intermittent and the signal to noise ratio is low. The topology of the field changes with time due to currents, wind, tides and storms. The field has a number of gateway nodes that have the capability to transmit off-field through a satellite, a ship or a plane.

We propose an approach to fusion that includes on-buoy processing, cooperative processing with nearest neighbors and the potential for off-field processing. Each stage of processing tries both to minimize false positive events and to maximize the probability of detection when an object is present. It also tries to minimize power used in order to prolong the life of the field.

We analyze the optimal placement of gateway nodes in the field to minimize power consumption and maximize reliability and probability of successful off-field transmission. We analyze the duty cycles of the sensor and gateway nodes to optimize lifetime. We also analyze the traffic that the field will be expected to handle in order to support network control and coordination, distributed fusion, off-field communication (including queries and responses, and reporting of detection events), forwarding of traffic through individual sensor nodes toward gateways or for fusion.
\end{abstract}

Keywords: Distributed sensor field, sensor networks, coastal surveillance

\section{INTRODUCTION}

The confluence of many technological advances, emerging needs, and innovation in architecture is driving towards viability and desirability of large and distributed sensor fields in a wide variety of applications. The applications may involve: short term and medium term weather prediction; understanding phenomena like tornados and hurricanes; detection and tracking of hidden and/or moving objects on ground, at sea, under sea, or in air; object tracking and targeting; detection of biological and chemical agents; etc. All these applications involve a large number of sensors, each collecting information, and use the collective information to achieve the goals of detection, tracking, targeting, etc. However, the applications differ in major ways. In particular, underlying physics of sensing, remote controllability of sensors, autonomous and uncontrollable movement of sensors, degree of collaboration needed and possible, possible ways to communicate results within and outside the field, energy constraints, and expected duration of use vary significantly among applications. Also, the applications differ significantly in the desirable trade-offs between false alarms and missed detection, in the penalty of the field itself being detected by others, in vulnerability to being fed wrong information, and in the value of the speed of detection. Thus, the design of sensor fields have to consider carefully the above factors associated with underlying application. Our research is aimed at such a comprehensive study and design methods for one specific category of sensor fields, one used to detect and track objects moving under water. The advent of low cost, low power, and small-size sensing and communication devices makes it economical to deploy large sensor fields for this application. On the other hand, the advances in quieting and hiding technologies and increasingly 'noisy' surroundings make it necessary to deploy a large number of closely spaced sensors to maximize detection probability while keeping the false alarm rate to a manageable level.

Much work has been done in the academic and industrial communities to develop sensing technologies for this application. Work has also been done in understanding the impact of some of the factors mentioned above.

(For further information, send correspondence to P.F. Chimento)

E-mail: Philip.Chimento@jhuapl.edu 
However, we wish to encompass in our work the collective influence of these factors on the design of topology and topology controls, design of communication architecture and protocols within the field and between the field and external world, design of fusion mechanisms within and outside the field. Our research is directed towards applying this research to the multi-dimensional problem in a coastal marine environment.

The problem we study involves 100 to 10,000 sensors placed in coastal waters. Sensors can communicate within the field using mobile ad hoc networking technologies. The communication level is small in absence of potential detection. However, it may increase significantly after a possible detection. Thus, false alarms may result in significant communication traffic and drain on the battery life. In many applications, batteries cannot be replenished and thus the false alarms create major reduction in the field lifetime. In order to minimize the probability of the field being detected and to minimize the drain on energy store in sensors, the sensors operate and communicate at low power and hence with short range. Thus, most pairs of sensors will need many hops to communicate with each other. Of course, some information needs to be communicated outside of the field to shipboard, airborne, or space-based platforms. This communication requires higher power and more expensive equipment, and hence the design of the field should use a limited number of nodes with external communication capability.

The environment introduces significant noise and creates a challenge in signal processing to achieve the right balance between false alarm and missed detection. A promising way to reduce the false alarm rate and its adverse impact on the lifetime of the field while maintaining a high probability of detection is to use intelligent fusion of information from many sensors and pass the results along. Besides improving the detection/false alarm ratio, the in-field fusion will help reduce the traffic within the field and from the field to outside. An important aspect of our work involves design of fusion architecture and algorithms, and investigating their efficiencies.

Some of the other factors with potentially significant impact on the design of the sensor field for our application are: uncontrollable movement of the entire field and of individual sensor nodes with respect to one another; random losses of sensor nodes; potential spoofing of some sensor nodes.

Our work will address the above factors in groups and in totality. The goal is to understand quantitatively the importance of various factors and combinations of factors in designing the sensor field for the type of application outlined here, and then focus on the critical subset of factors to develop an design approach and algorithms.

The current paper is an overview paper that describes the model in some detail, motivates and describes the factors to be studied. We also provide quick look analysis of some of the factors, discuss trade-offs, and identify trade-offs that need further investigation. We also describe potential approaches to in-field fusion and provide initial analysis. Future papers will discuss details of simulation and analytic models, quantitative results, and design approaches and algorithms.

\section{MODEL}

The sensor fields we consider typically involve $N=100$ to 10,000 sensor nodes. The configuration depends on the area to be covered for detection. Initial deployement may be in a rectangular grid or it may take an irregular shape conforming to the local geography. For simple analysis, we will model it using a square planar grid. Simulation models will consider more complicated topologies.

Detection is based on reflection phenomenon. That is, a sensor sends a burst of radiation (ping) and all sensors listen. An object of interest will reflect and provide an ellipse of uncertainty to each listener. The parameters of the ellipse are governed by the object to be detected and by the distances between the sensors. Of course, the signal received may be affected by undersea as well as surface environmental phenomena. The noise may be stronger near the shore than at sea. Of course, if the signal from multiple sensors can be shared, then the collective information may have better signal to noise ratio. Sensors consume energy in pinging and listening. Thus, the number of pings during a sensor's life time is limited. The frequency and order of pings may be preprogrammed or it may be decided in a distributed fashion by rules based on recent pings in the neighborhood.

We assume that the sensor-sensor communication uses power $P$, giving a nominal range $R$ and nominal data link rate $D$. Of course, these parameters change depending on the environmental noise, fading, and other 
impairment. The sensor-sensor communication may be used to communicate results to other sensors so they can correlate the results and use fusion to improve detection probability and reduce false alarm probability. Sensor-sensor communication is also used to move the information to gateways capable of communicating to external nodes. We assume that the normal sensor nodes have a total supply, $E$, of energy available. There are $N_{g}<N$ gateway nodes. These nodes have normal sensor functionality plus additional energy supply of $E_{g}$ in the equipment designed for external communication (to ships, planes, satellites, etc.). Both the number $N_{g}$ and the location of these $N_{g}$ nodes are design variables.

Our sensor field may involve one or two more types of special nodes (nodes that do more than basic sensing and communication with other sensor nodes) in addition to the gateway nodes:

The second set consists of fusion nodes. These nodes get sensing results from nearby sensor nodes and apply fusion algorithms to derive a composite picture. The results, if of interest, are sent to the nearby gateway node for communication to an external node where further fusion can be accomplished and action can be taken. The results can also be used to impact local operation of the field (ping order and interval, power usage, additional fusion, etc.). Fusion may help improve and reduce false alarms. It may also help reduce traffic to gateways and to external nodes. The number of fusion nodes, $N_{f}$, and their locations are design variables. If $N_{f}$ is zero, we have no fusion inside the field and fusion, if any, is done externally.

The third type of special node is a cluster head. For large fields (large $N$ ) and small range $(R)$, some sensor-sensor communication will involve a large number of hops using many sensor nodes acting as relays. If we have a small number of gateways, then communication from a sensor to a nearby gateway may use many hops and hence use energy from many sensor nodes. The busiest nodes will be the ones near the gateways. Clustering is one way to mitigate the imbalance in loading created by a large number of hops in communication. Effectively, a hierarchy is created among sensor nodes. Some nodes are able to communicate longer distances among themselves with higher power (and possibly at a different frequency). These nodes will also communicate with normal nodes at normal power. These nodes are called cluster heads. Sensor-sensor communication and sensor-gateway communication may involve sensor-sensor-cluster head-cluster head-gateway type routing. By using cluster head network as a higher layer backbone, normal nodes are relieved of some of the communication burden. Once again, the number and locations of cluster heads are design variables. Of course, there may be close relationship between fusion nodes, cluster heads, and gateways. Cluster heads may carry an extra energy supply of $E_{c}$. There is also a possibility of designing the field so every node can become a cluster head. In that case, cluster head configuration can be changed dynamically based on the field status or periodically.

In addition to the topological network design described above (and the various overlay networks involved), communications protocols for the field that are appropriate to the environment and the application must be chosen from ones already developed or newly designed. Key protocols that must be determined and modeled are: MAC (layer 2) protocols and routing (layer 3) protocols. In addition, gateway selection, fusion node selection, cluster head election, cluster head routing and fusion (application layer) protocols are needed.

Influencing all of these protocols are energy and environmental considerations, the physical layer chosen and the behavior of the application itself. The mission of the sensor field will determine how to optimize the design trade-offs that we must make in these designs.

As mentioned above, one of the key features in our model of the distributed sensor field is the ability to apply fusion algorithms at a subset of nodes, send fused information out to external nodes, and even modify sensor field behavior based on fused information. The approach to fusion and the algorithms used are likely to depend on the underlying physics and may use some learning techniques.

\section{FUSION APPROACHES}

Properly designed fusion techniques can combine observations from multiple sensors that are spatially distributed to reduce false alarms while maintaining the required level of detection probability. For the coastal water surveillance problem studied here, fusion algorithms can be applied to the detection, localization, tracking, and also the formulation of dynamic sensor control policy after a detection. Following the standard JDL data fusion model and its revisions, ${ }^{1-3}$ the fusion problems considered here fall under "Level-One Processing" (Object Refinement) and "Level-Four Processing" (Process Refinement, necessary for the active sensor control). In this 
paper we focus our discussion on the detection problem. Two unique issues that set the particular fusion problem for coastal water surveillance apart from the traditional data fusion are:

- The importance of in-network fusion driven by power constraints. The underwater acoustic environment in the littoral space is characterized by a large amount of clutter that can result in a significant number of false alarms for any single sensor node. These false alarms can trigger a large amount of unnecessary communications to the gateway nodes and hence lead to fast depletion of power available at the bottleneck sensor nodes. Proper fusion of sensor observations within the network to reduce false alarms transmitted through the gateway nodes is critical if the network is expected to operate autonomously over an extended period of time. This notion of in-network fusion is distinct from the typical fusion-center-based notion considered in the data fusion or sensor network literature.

- The extensive coverage of the sensor buoy field relative to the range of the target signal. Given the significant issue of clutter in the littoral space, a sensor needs to be located "close enough" to the source of the target signal to ensure high enough signal-to-noise ratio to achieve acceptable local detection performance. Equivalently, this defines a detection range for the target that can be significantly smaller than the coverage of the entire sensor field. In other words, given a target with a specific location, only a portion of sensor nodes are statistically relevant for the detection of the target. Therefore, arbitrary inclusion of observations from sensors spreading beyond the detection range in a fusion could potentially lead to degradation of performance. This is in contrast to the typical model assumed in the literature on decentralized detection for sensor networks where fusion is performed on the observations collected over the entire network (see, for example $\left.{ }^{4,5}\right)$.

Taking into account the above unique requirements, we characterize a fusion approach based on a "multilayered" architecture that permits both in-network fusion and fusion-center-based fusion. Each in-network fusion is performed on information from a set of sensor nodes within the network (for example, at a pre-designated or dynamically elected sensor node); the result of in-network fusion is then transmitted to a fusion center, located outside the sensor field, through gateway nodes. We will refer to the nodes where in-network fusion is performed as fusion nodes. It is possible that multiple layers of in-network fusion are performed before the fused information reaches the fusion center. Such a multi-layered architecture enables us to analyze the trade-offs between the expected fusion performance and power efficiency.

The fusion algorithms, either for in-network or fusion-center-based approaches, determine the type of information that needs to be exchanged and the anticipated performance for detection. Fusion algorithms can be classified into three broad categories:

- Observation-level fusion: The signals observed by the sensors, after basic signal processing (for example, filtering, digitization, etc.), can be combined to extract relevant information. Examples of such fusion include localization techniques for acoustic sources and beamforming. ${ }^{6,7}$ Such fusion techniques, when the underlying models are well understood, can lead to significant performance gains. However, we argue that such techniques are not appropriate for in-network fusion in a buoy field due to requirements for synchronization and communications among the sensor nodes. In order to support coherent processing techniques, we need to ensure tight frequency, phase, and timing synchronization across sensor nodes. Drifting buoys makes this a challenging task. Moreover, the data rates required for sending the observations to the fusion nodes can be very high and hence consume significant power. It is possible that each buoy can carry multiple sensors, and beamforming fusion can be applied to combined observations from these sensors at the buoy.

- Feature-level fusion: Each sensor node can first process the local sensor observation to extract relevant features (for example, the strength and the direction of the detected signals or other sufficient statistics) before transmitting the features to a fusion node for fusion. The data rate requirement is moderate comparing with the requirement for the observation-level fusions. 
- Decision-level fusion: When the communication or power resources are scarce, each sensor node can apply algorithms locally to reach a decision (for example, a binary detection decision) and then transmit the local decision to a fusion node for fusion. Examples of decision-level fusion algorithms include the simple voting scheme, Bayesian methods, and other decentralized detection algorithms. ${ }^{4,8}$ The communication requirement for the decision-level fusion is minimum. At the same time, the potential performance gain can be limited. Recent results ${ }^{5,9}$ on decentralized detection in sensor networks suggest that fusion of binary decisions from identical sensors is asymptotically optimal when resources for communications are constrained. However, these results are established under a strong i.i.d. assumption and the fusion-centerbased model (all the sensor nodes are fused).

We will consider both the feature-level and decision-level fusion algorithms when investigating the trade-offs between fusion performance and power efficiency under the multi-layered fusion architecture discussed above.

\subsection{An illustrative example}

In order to study the trade-offs between the fusion performance and power efficiency, we will need to characterize the expected performance of the fusion algorithms as a function of the relevant design parameters. Following the proposed multi-layered fusion architecture, the relevant parameters include the number of fusion nodes $N_{f}$, the extent of each fusion (the set of sensor nodes participating in the fusion), and the specific fusion techniques used in each fusion. Accurate characterization of fusion performance will require detailed models of the sensor, target signal distribution, clutter distributions, and might ultimately require proper Monte Carlo simulations. Here, we provide a simple analytical model for distributed detection to illustrate the type of trade-offs we will consider via simulations under more realistic assumptions.

Let us assume that the number of fusion nodes is large enough so that the sensor nodes sending information to each fusion node does not cover a large area relative to the effective range of the target signal. Furthermore, we will assume that sensor observations included in each fusion are i.i.d. given the hypothesis*. To understand the fusion performance as a function of the number of participating sensor nodes without making additional assumptions about the distribution of sensor observations (for example, Gaussian), we consider the simple decision-level fusion based on voting. Specifically, let $m$ be the number of sensor nodes participating in a fusion; $m=N / N_{f}$ if we assume each node only participate in one in-network fusion. Assume that the performance of the local detectors are identical and is characterized by the detection probability $P_{D}$ and the false alarm probability $P_{F}$. Then the false alarm probability after the fusion, denoted by $\bar{P}_{F}$ at the fusion node can be derived as

$$
\bar{P}_{F}=P\left\{N_{1}>\left\lceil\frac{m}{2}\right\rceil \mid H_{0}\right\}
$$

where $N_{1}$ is the number of nodes with positive detections.

Under the independence assumption, we can obtain bounds for $\bar{P}_{F}$ by applying the Hoeffding inequality ${ }^{10}$ :

$$
\bar{P}_{F} \leq e^{-2\left(0.5-P_{F}\right)^{2} m} .
$$

Note that the bound obtained using the Hoeffding inequality, as shown in (2), is tight only for large $n$. As illustrated in Figure 1(c), the false alarm probability decreases slowly as the number of fused nodes increases beyond some point. The number of false alarms is directly related to the amount of traffic transmitted to the gateway nodes. As the number of fusion nodes $N_{f}$ increases (the number of fused nodes decreases), the communications to the gateway could increase driven by the higher false alarm probability $\bar{P}_{F}$. On the other hand, a small number of fusion nodes translates into a large number of fused nodes and hence more communications to the fusion nodes. Combing this analysis with the analysis on power consumption for communications will enable us to properly trade off the fusion performance with the power efficiency.

\footnotetext{
${ }^{*}$ We recognize that this assumption is unrealistic if the sensor nodes involved in a fusion spread over a wide area. Nevertheless, this assumption is made throughout the literature on decentralized detection
} 


\section{COMMUNICATION SUBSYSTEM}

The communication subsystem is highly dependent on the design of the rest of the surveillance system. Many of the trade-offs that have to be made will be driven by the application requirements. In this section, we outline some of the options for communication subsystem components and how the trade-offs will be analyzed.

\subsection{Network Traffic}

Sensor network traffic both affects and is affected by the choices of protocols for communication within the field, as well as by the design of application processing. In this subsection, we describe briefly the different types of traffic that the communication subsystem in the sensor field must handle. We can classify traffic sources as follows: Sensor (application) sources; query sources and responses; and network control sources. In part, the traffic generation patterns of the field will depend upon the types of surveillance targets; surface targets will present different signal characteristics to the sensors than underwater targets, as well as have different movement patterns and speeds. In general, though, we can expect low signal-to-noise ratios at the sensors and consequently a high false positive rate. We assume that initial sensor contact messages will be fairly frequent, but because we also assume significant in-field processing, we expect this traffic to be distributed only locally among nearest neighbors (i.e. 1 or 2 hops away) and not sent to the gateways. This local traffic may be directed toward fusion nodes that have additional processing capabilities, or may trigger local distributed processing in order to provide initial false positive filtering.

We envision that the application-driven traffic patterns will evolve in the following way: As a result of an active probe (for example a "ping") or as a result of passive monitoring, the sensors on an individual node determine a signal of interest. To determine whether the signal is truly significant, the sensor node shares its information with neighboring nodes which, in general, should also have sensed a signal. (Note that environmental phenomena may cause signals to be "heard" far beyond their normal propagation range.) The sensor nodes collaborate locally and determine whether the information from the neighborhood of sensors requires further processing and fusion. At this point, one or more of the sensors may forward this information to fusion nodes. Note that an alternate design is to skip the local processing and always simply forward information to fusion nodes for processing.

The fusion nodes, having more processing power and information from multiple sensor nodes, determine whether the event sensed by individual sensors should be sent off-field. In this case, the fusion nodes (or the individual sensor nodes) forward their information to gateway nodes for off-field transmission. Note that in this system model, we have $N \geq N_{f} \gg N_{g}$ where $N$ is the number of sensor nodes, $N_{f}$ is the number of fusion nodes and $N_{g}$ is the number of gateway nodes.

The types of traffic patterns that emerge from this behavior are: scoped local broadcast (for initial information sharing) and many-to-one unicast (for sensor node to fusion node communication and for fusion node to gateway node communication). We expect the scoped local broadcast to have the frequency of false positives, and the frequency of the many-to-one unicast communication to be somewhat less; that is, some fraction of the false positive rate corresponding to the reduction in false positives expected from local processing. We also expect that on average, the length of the paths to the fusion nodes will be much shorter than the length of the paths to the gateway nodes.

Another type of traffic that the sensor field will have to handle is query/response traffic. This traffic is initiated from outside the sensor field, possibly in response to fused information sent from the field through the gateways. We assume that queries of individual nodes, as well as groups of nodes is possible, and further that these queries may produce relatively large quantities of information in response, perhaps even megabytes if the query is for recent historical (i.e. time-series) data from the sensors.

Queries may target individual fusion nodes, groups of fusion nodes or geographical neighborhoods of sensor nodes. These different query types produce (respectively) unicast from the gateway to a fusion node and back; multicast from the gateway to fusion nodes and many-to-one unicast patterns back from the fusion nodes to the gateways; limited multicast from the gateway to sensor neighborhood and many-to-one unicast patterns back to the gateway. We assume that the query messages are short, but the unicast responses are relatively large and comprise multiple messages. This type of communication holds the greatest potential for congestion. 
By "network control sources" we mean traffic from the protocols that maintain the communication infrastructure of the sensor field. This includes especially routing protocols and gateway- and fusion-node election protocols in addition to clustering protocols. This traffic is most sensitive to the choice of protocol and to sensor field movement.

We expect that as a coastal surveillance field is deployed, that significant drift will occur through the lifetime of the field. As nodes drift into or out of radio range of one another, the communication topology changes and consequently routing information and information about the nearest specialty node (i.e. gateway, fusion or cluster head node) and cluster identity will change. Because the topology is so dynamic, we think that path determination is best done as late as possible before packets are sent. The same strategy holds for determining specialty nodes.

This observation argues for reactive rather than proactive routing protocols. It also argues that route caches and caches of the closest specialty node and cluster identification information have timers that are tied to the drift rates expected for the field. However a negative consequence of this approach is that before communication takes place after there has been a pause, there will be a burst of network control traffic to determine the closest fusion and gateway nodes and to determine routes to these nodes. Typically these will involve multicast and broadcast from nodes wishing to communicate and unicast return traffic. Although in large sensor fields this is unlikely to cause widespread congestion, there is potential for local congestion and delay before communication can be sent. All of these issues will be modeled and reported upon in a subsequent paper.

\subsection{Gateway/Fusion Node Planning}

As mentioned in the Section 2, we assume that a small subset of the sensors covering the sensor field $\left(N_{g}\right.$ out of $N$ ) is equipped with special communications capability to communicate with nodes outside of the sensor field. These sensors play the role of gateways for off-field communication in the sense that all communications intoor out of the field is through these nodes, and the other non-gateway nodes are only capable of sensor-to-sensor communication. Figure 1(b) shows a sensor field with three gateways using a satellite for off-field communication. This allows for a lower cost design by concentrating expensive communication devices in a small subset of the nodes. This subsection discusses an important problem in designing such gateway-based sensor networks. That is, determining the number of gateway nodes needed and their location in the sensor field. We will show that, in determining the number of gateways, the tradeoff is between performance and cost. As more gateways are deployed, less traffic load is placed on each gateway and its surrounding nodes, resulting in longer network lifetime. However, with a larger number of gateways the network may be too costly to deploy since gateway nodes are more expensive than non-gateway sensor nodes.

Consider the square planar grid shown in Figure 1(a) made up of one gateway and $n$ sensors. In this grid it is assumed that the radio range $R$ is such that each non-boundary sensor has four neighbors within range. It can be easily shown that in such grid the number of sensors that are $h$ hops away from the gateway is $n_{h}=4 h$ and $n=2 H(H+1)$ where $H$ is the largest hop count (associated with the boundary nodes). If each node generates sensor data destined for the gateway at a rate of $r$ messages per unit time, it can be shown that a node $h$ hops away from the gateway will need to transmit messages at a rate

$$
R_{h}=\left[\frac{H(H+1)}{2 h}-\frac{h-1}{2}\right] r=\left[\frac{n}{n_{h}}-\frac{h-1}{2}\right] r .
$$

Figure 1(d) is a plot of $R_{h} / r$ as a function of $h$ for different values of $H$. This figure shows that most of the message forwarding load is placed on the first-hop sensors (sensors that are located one hop from the gateway). Since radio communication is the main source of energy consumption, the network lifetime will be determined by the lifetime of these first-hop sensors, and we will proceed with a first-hop analysis to determine the number of gateways needed to achieve a given lifetime for the sensor field.

The forwarding rate $R_{1}$ at the first-hop nodes is made up of two components

$$
R_{1}=\left(\frac{n}{n_{1}}\right) r=\left(\frac{n}{n_{1}}-1\right) r+r
$$


with the first term corresponding to relayed traffic (received from two-hop nodes) and the second term corresponding to traffic generated from local sensing. Let $e_{t}$ denote the energy consumed to transmit a message and $e_{r}$ the energy consumed when relaying a message, which includes receiving the message from a neighbor and transmitting it to a next-hop neighbor. Obviously, $e_{r}>e_{t}$, with $e_{r}-e_{t}$ reflecting the energy dissipated during the message receive phase. With $E$ being the initial energy supply at each sensor (at time $t=0$ ), the lifetime of a first-hop sensor corresponds to the time $T$ when the total dissipated energy is equal to $E$ :

$$
\left(\frac{n}{n_{1}}-1\right) r T e_{r}+r T e_{t}=E
$$

and is given by

$$
T=\frac{\frac{E}{r}}{\left(\frac{n}{n_{1}}-1\right) e_{r}+e_{t}} .
$$

In order to derive the sensor network lifetime as a function of the number of gateways $\mathrm{Ng}$, consider the following:

- As we discuss below, the sensor field will be organized into a number of gateway areas (one per gateway) and each sensor will be associated with a gateway in such way that the overall communication load is balanced among the gateway areas, which is achieved when $n=N / N_{g}$. Consequently, first-hop sensors associated with different gateways are expected to have similar lifetimes.

- Even if each gateway area is not a square grid as in the model of Figure 1(a), the first-hop analysis remains valid as long as the loads on each of the first-hop nodes in an area are comparable, and even when the number $n_{1}$ of such nodes is different from the regular grid case where $n_{1}=4$.

Substituting $n=N / N_{g}$ in the above equation we obtain

$$
T=\frac{\frac{E}{r} N_{g}}{e_{r} \frac{N}{n_{1}}-\left(e_{r}-e_{t}\right) N_{g}},
$$

which is shown graphically in Figure 1 (e) for the case $N / n_{1}=10^{3}, e_{r} / e_{t}=1.2, E / e_{t}=10^{6}$, and $r=0.1$ message/sec. For small values of $N_{g}$, which is the case in practical situations (the operational range in Figure 1(e) is limited to $\left.N_{g}<N / n_{1}\right), T$ is essentially linear in $N_{g}$. Given a target lifetime $T$, the number of gateways can be determined from equation 6 .

Note that so far we assumed that the selection of the number of gateways is driven more by network lifetime considerations than by network capacity considerations. Obviously, the aggregate bandwidth that can be achieved out of the sensor field increases with the number of gateways, and if capacity could be limiting then a similar analysis can be done to determine the minimum number of gateways needed. However, with enough fusion being performed inside the network, it is more likely that the number of gateways will be driven by lifetime and fault tolerance rather than bandwidth (capacity) considerations. Since gateway nodes are more expensive than sensor nodes, network cost could also play a role in the sense that the number of gateways could be constrained to less than some limit $N_{g}^{\prime}$. In this case, whether the lifetime objective is met depends on whether the lifetime corresponding to $N_{g}^{\prime}$ is at least as large as the target one. If not, then the achievable lifetime will be limited to $T\left(N_{g}^{\prime}\right)$.

Once the number of gateways is determined, the next step in planning the sensor network deployment is to determine where gateways need to be placed. Since the initial energy allocation is the same among the sensors, the gateways need to be placed in such a way that the load is balanced among the different gateway areas, resulting in first-hop sensors in all areas running out of energy at the same time. Under uniform density, the sensor field is partitioned into areas of similar size with one gateway at the center of each area as shown in Figure 1(b) for an example with three areas. As we discuss in the next subsection, to enforce this partition during network operation, a protocol for automating the sensor-to-gateway association is needed. 


\subsection{Topology Control and Routing}

In a dense sensor network, it is more energy efficient to select a sparser topology for radio communication. Given the set of sensors $V$, the transmission topology is determined by the graph $G=(V, E)$ where $E$ is the set of edges between any two nodes in $V$ that can communicate when using maximum power. The role of topology control is to conserve energy by computing and maintaining a connected topology $T$ which is a sub-graph of $G$. Some of the approaches used include power control and relay node control.

With power control, all nodes keep their radio modules operating but with different power levels determined by topology control. By lowering power, some links in $E$ are removed resulting in $T=\left(V, E^{\prime}\right)$ where $E^{\prime}$ is a subset of $E$. There are a number of studies in this area; they typically use some radio propagation model and assign node power to generate a topology with some bound on the maximum node degree or on the energy stretch factor, defined as the maximum over all node pairs $u$ and $v$ of $E_{G}(u, v) / E_{T}(u, v)$ where $E_{G}(u, v)$ (respectively $E_{T}(u, v)$ ) denote the energy of the minimum-energy path between $u$ and $v$ in $G$ (respectively in $T$ ). ${ }^{11,12}$ Under relay node control, a subset of the nodes are identified as relay nodes and form a connected topology $T$ among them $\left(T=\left(V^{\prime}, E^{\prime}\right)\right.$ where $V^{\prime} \in V$ and $\left.E^{\prime} \in E\right)$, the non-relay ones are considered redundant for the purpose of packet forwarding and can be placed in a communications sleep mode (by turning off their radios). A dynamic topology control protocol is used so that nodes can alternate going into this sleep mode in order to balance energy usage among them. Cluster-based protocols use active neighbor discovery so that nodes can group themselves into clusters identified by a cluster-head with packet forwarding handled by these cluster-heads, and nodes take turns being cluster-heads. ${ }^{13}$ Cluster heads may use different frequencies than sensors for communication.

The sparser topology $T$ (with a smaller number of nodes and/or links) identified by topology control is the one used by the routing protocol for path selection (in the extreme case when $T$ is a strict spanning tree no path selection is needed). There are a number of proposals for routing in wireless ad-hoc networks (see ${ }^{14}$ for a survey of routing in sensor networks and ${ }^{15,16}$ for general discussion of issues that could affect routing). They belong to one of two main categories: proactive or on-demand routing. Proactive routing attempts to keep routing information for all the nodes up to date by advertising topology changes. Each node maintains routes to all reachable destinations at all times, whether or not there is currently any need to deliver packets to those destinations. However the size of the network and the mobility of the nodes present a big challenge. In contrast to proactive algorithms, reactive routing protocols discover routes only when they are needed by flooding routerequest messages in the network, these routes are cached and updated on-demand. Hybrid protocols have been proposed that maintain up-to-date routing information about destinations within some neighborhood and use on-demand routing for distant nodes.

As discussed in Section 4.2, the existence of multiple gateways in the field and the potential difference in target detection activity in different gateway areas (and the resulting uneven energy consumption) calls for a dynamic sensor-to-gateway association protocol (DS-GAP). The DS-GAP is in addition to any topology control and routing mechanisms that may be in place, but these three components can be closely related and intertwined.

While the presence of multiple gateways offers extended lifetime and a higher degree of off-field communication reliability, a sensor will need to select one of the gateways at a time for off-field communication. Based on attributes that include current available energy levels, the distributed protocol dynamically assigns sensors to gateways in such a way that the overall load is balanced among the different gateway areas over the lifetime of the sensor field. The gateways will need to advertise themselves; and sensors choose one of the gateways as their destination gateway. In the uniform density case, choosing the closest gateway (as measured by the minimum hop path to the gateway) should result in the desired partition into gateway areas of similar size. However, this is not most efficient because (1) the sensor field density may not be uniform, and (2) over time the available energy will not be the same in different areas due to different target detection activities. A DS-GAP is needed to reassign sensors among areas when needed to equalize the available energy in the different areas, resulting in extended network lifetime. With such a protocol, the partition into gateway areas will change over time with area sizes expanding or shrinking as required for load balancing. Gateways will need to advertise available energy, which is dominated by the available energy in their first-hop sensors. First-hop sensors will

have to inform the gateway of their available energy, this could be done systematically by all nodes as part of their periodic neighbor-to-neighbor hello message exchange. Gateways will then periodically flood a message to all sensors indicating among other things the available energy level. As these messages are relayed they can 
also record path information such as hop count. A sensor receiving these messages from different gateways can select the most appropriate gateway taking into account advertised available energy and possibly other attributes such as hop count. Flooding of these gateway messages can take advantage of an underlying topology control mechanism where only a subset of the nodes will need to relay the messages. Once a sensor selects a particular gateway, it can use an existing routing protocol to route its messages to the selected gateway. Alternately, it can forward messages along the reverse path that the gateway advertisement message was forwarded on (if such path information is maintained). Details about the DS-GAP operation and its performance evaluation will be communicated in another paper.

In Section 2, we also mentioned another set of special nodes, namely fusion nodes. However, the presence of these nodes and fusion mechanism also have an impact on the traffic pattern and this effect will play a role in the design of gateway and cluster topologies. In particular, fusion may reduce the size of messages to be sent beyond the fusion node towards the gateway. It will also consolidate information from many sensors to a single message from the fusion node to the gateway. This will help the nodes between fusion node and the gateway in reducing energy consumption and may even create a more balanced energy consumption pattern. Fusion will also help reduce the false alarm rate and hence reduce major drain on the energy store. Since the magnitude of the combined impact depends on the specifics of fusion, the traffic impact and impact on overall topology will be discussed in a future paper.

\subsection{MAC Protocols}

It is generally known that in sensor networks, radios are the largest consumers of energy. The choice of MAC protocol is one of the keys to the energy efficiency of the sensor field.

As we focus on the particular characteristics of our application and on the traffic characteristics identified in Section 4.1 we find that we have a number of requirements of a MAC protocol. Because of local multicast requirements, both for initial filtering and fusion, as well as for group queries and for route determination by routing protocols, several nodes in a neighborhood may attempt to use the medium nearly simultaneously. For this use, contention resolution must be fast and all the nodes that have signal information to distribute to neighbors should get access to the medium. The efficient re-use of the medium is important in this case, so that there are not long and multiple contention and backoff periods between transmissions.

When the sensor nodes or fusion nodes are transferring the results off-field or responding to queries, there is potentially a large amount of information that must be sent from multiple nodes to a gateway or a single point in the network. In this case, the relay behavior of each node is important along with the fact, pointed out in Section 4.2, that for nodes closer to the destination, more and more transit traffic must be handled. This implies that the MAC protocol must have fairness properties when there is high demand for the medium. Otherwise traffic from one or more routes may be dropped, causing those routes not to be "equal cost" to the system.

Another characteristic of the MAC protocol that is necessary for our application is that it minimizes loss and consequently retransmissions. Again, in the communication mode where a query response is being sent or the identification of a possible surveillance target is being reported, dropping a packet is not necessarily a problem in terms of latency and reliability at the application layer. For example, increasing latency by a few seconds will not make much difference when a target is moving at 40 knots - it represents a difference in position of only 20.58 meters per second. In addition, packet loss can be compensated by Layer 4 protocols. The real costs to the sensor field in our application are in energy and throughput. Every dropped packet represents not only a waste of energy for the node that drops it, and waste of capacity on the local medium, but it also represents a waste of both nodal energy reserves and medium capacity summed over the entire path that the packet took up to the point of dropping. Consequently, the MAC protocol for our application should be drop-averse; the same characteristic applies to the routing protocol. In fact, the longer that a packet has traveled through the network (in terms of hop count) the harder it should be to drop it.

In summary, then, the MAC protocol for our application should be energy efficient, drop averse, fair, support relay efficiently, and allow fast, efficient contention resolution.

Much of the published work on MAC protocols for sensor networks has focused on 802.11-like protocols that are based on contention, back-off and some form of sleep-duty-cycle that allows for good energy efficiency. Good 
examples of this approach are S-MAC ${ }^{17,18}$ and T-MAC ${ }^{19}$ though there are many others. However, there are other approaches, such as $\mathrm{LEACH}^{20}$ and $\mathrm{SCR}^{21}$ that use hybrid approaches (e.g. clustering, CSMA and TDMA for the former and contention signalling and TDMA for the latter) that look promising for our application. Our future work in this area will consist of evaluating these approaches in light of the required MAC characteristics developed in this section.

\section{ACKNOWLEDGMENTS}

We would like to acknowledge the many discussions and contributions of our colleague Chris Diehl to this work.

\section{REFERENCES}

1. F. E. White, "A model for data fusion," in Proceedings of the 1st National Symposium on Sensor Fusion, 1988.

2. A. Steinberg, C. Bowman, and F. White, "Revisions to the JDL data fusion model," in Proceedings of the SPIE Sensor Fusion: Architecture, Algorithms, and Applications, 3719, 1999.

3. D. L. Hall and J. Llinas, Handbook of Multisensor Data Fusion, CRC Press, Boca Raton, 2001.

4. R. Niu, P. Varshney, M. Moore, and D. Klamer, "Decision fusion in a wireless sensor network with a large number of sensors," in Proceedings of the Seventh International Conference on Information Fusion, $\mathbf{1}$, pp. 21-27, (Stockholm, Sweden), June 2004.

5. J.-F. Chamberland and V. V. Veeraalli, "Asymptotic results for decentralized detection in power constrained wireless sensor networks," IEEE Journal on Selected Areas in Communications 22, pp. 1007-1015, August 2004.

6. J. Chen, L. Yip, J. Elson, H. Wang, D. Maniezzo, R. Hudson, K. Yao, and D. Estrin, "Coherent acoustic array processing and localization on wiireless sensor networks," Proceedings of the IEEE 91, pp. 1154-1161, August 2003.

7. G. Barriac, R. Mudumbai, and U. Madhow, "Distributed beamforming for information transfer in sensor networks," in Proceedings of the 3rd International Symposium on Information Processing in Sensor Networks (IPSN'04), (Berkeley, CA), April 2004.

8. J.-F. Chamberland and V. V. Veeraalli, "Decentralized detection in sensor networks," IEEE Transactions on Signal Processing 51, pp. 407-416, February 2003.

9. G. Polychronopoulos and J. N. Tsitsiklis, "Explicit solutions for some simple decentralized detection problems," IEEE Transactions on Aerospace and Electronic Systems 26, pp. 282-292, March 1990.

10. W. Hoeffding, "Probability inequalities for sums of bounded random variables," Journal of American Statistical Association 58, pp. 13-30, 1963.

11. V. Rodoplu and T. Meng, "Minimum energy mobile wireless networks," IEEE Journal on Selected Areas in Communications 17, pp. 1333-1344, August 1999.

12. R. Wattenhofer, L. Li, P. Bahl, and Y. Wang, "Distributed topology control for power efficient operation in multihop wireless ad hoc networks," in Proceedings of INFOCOM 2001 Volume 3, pp. 1388-1397, IEEE, IEEE, April 2001.

13. Y. Xu, S. Bien, Y. Mori, J. Heideman, and D. Estrin, "Topology control protocols to conserve energy in wireless ad hoc netowrks," tech. rep., USC/ISI, 2003.

14. J. Al-Karaki and A. Kamal, "Routing techniques in wireless sensor networks: a survey," IEEE Wireless Communications 11, pp. 6-28, December 2004.

15. I. Akyildiz, W. Su, Y. Sankarasubramaniam, and E. Cayirci, "Wireless sensor networks: A survey," IEEECommunications Magazine 40, pp. 102-114, August 2002.

16. K. Romer and F. Mattern, "The design space of wireless sensor networks," IEEE Wireless Communications 11, December 2004.

17. W. Ye and J. Heidemann, "Medium access control in wireless sensor networks," Tech. Rep. ISI-TR-580, USC/ISI, 2003.

18. W. Ye, J. Heidemann, and D. Estrin, "Medium access control with coordinated, adaptive sleeping for wireless sensor networks," Tech. Rep. ISI-TR-567, USC/ISI, January 2003. 


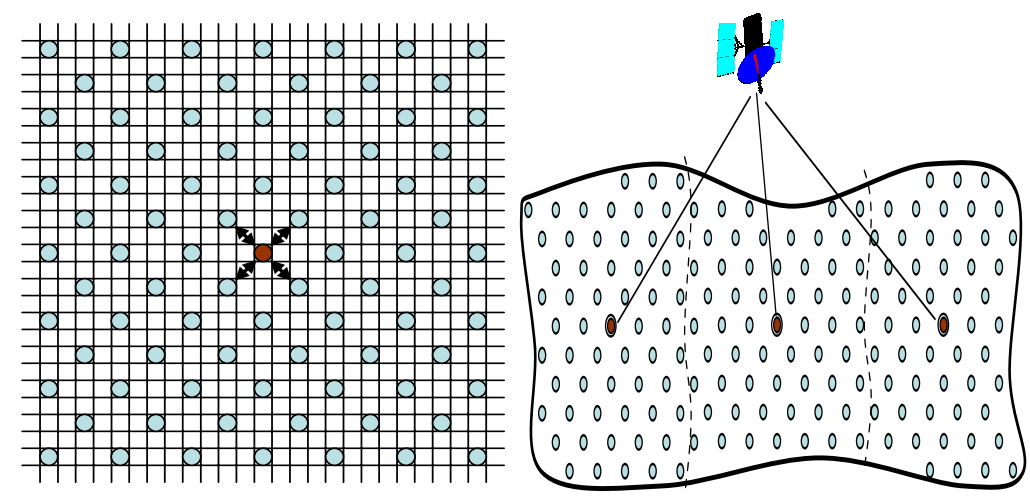

(a) A uniform grid sensor field

(b) Sensor field with three gateways

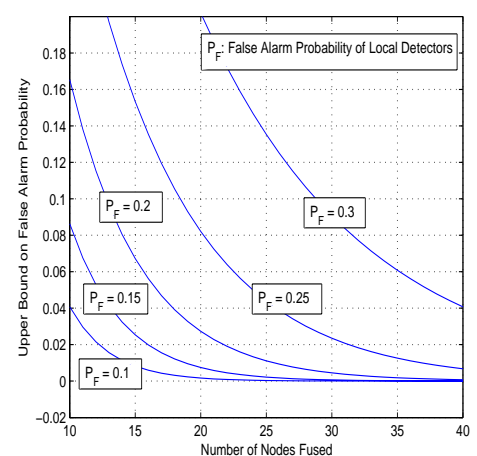

(c) Upper Bound on the False Alarm Probability

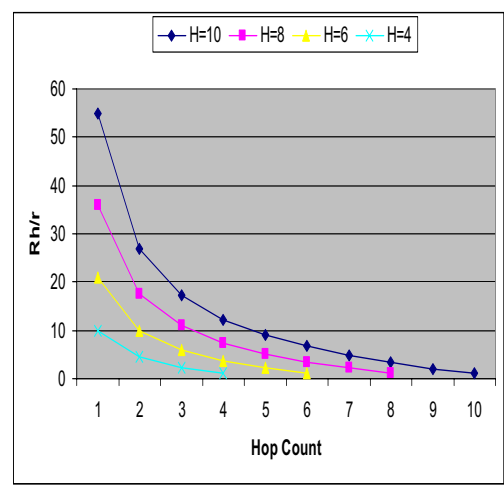

(d) Forwarding rate as a function of distance from gateway

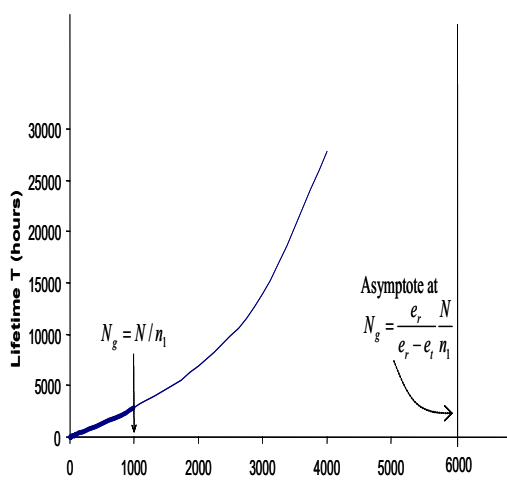

(e) Network lifetime as a function of number of gateways

19. T. van Dam and K. Langendoen, "An adaptive energy-efficient MAC protocol for wireless sensor networks," in SenSys '03, ACM, November 2003.

20. W. Heinzelman, A. Chandrakasan, and H. Balakrishnan, "Energy-efficient communication protocol for wireless microsensor networks," in Proceedings of the 33rd Hawaii International Conference on System Sciences, IEEE, IEEE, 2000.

21. J. Stine, "Integrating the physical and link layers in modeling the wireless ad hoc nettworking MAC protocol, synchronous collision resolution (SCR)," Tech. Rep. Mitre, Mitre Corporation, September 2003. 